\title{
Robotic Automated External Defibrillator Ambulance for Emergency Medical Service in Smart Cities
}

\author{
Nasir Ahmad \\ Sr. Lecturer, Department of ECE, Sankar Polytechnic College, Tirunelveli, Tamil Nadu, India
}

\begin{abstract}
Robotic systems are one of the key solutions for providing smart services. Time is a critical issue when dealing with people who experience a sudden cardiac arrest that unfortunately could die due to inaccessibility of the emergency treatment. Therefore, an immediate treatment using Automated External Defibrillator (AED) must be administered to the victim within a few minutes after collapsing. Hence we have designed and developed the Ambulance Robot, shortened as Ambubot, which brings along an AED in a sudden event of cardiac arrest and facilitates various modes of operation.The prototype with biomedical sensors are used for monitoring the patient health continuously, the location of the patient can also be tracked in case of emergency via gsm.
\end{abstract}

KEYWORDS: ECG Sensor, IR Sensor, Pic Controller, Gps/Gsm, LCD

\section{INTRODUCTION}

The concept of high-tech machines that can serve the people well or relieve humans of tiresome chores has been an object of human imagination. A mobile robot would be able to travel throughout the environment and can put their position wherever its condition. Mobile robot is an autonomous or semi autonomous machine that capable to move around in their environment and also can perform various tasks either with direct or partial control by human supervision or completely autonomous. With using multiple sensors for navigation, this robot is able to navigate from a point to a given destination without losing the correct path or hitting obstacles. the system uses an transducer and a microprocessor-based circuit to record blood pressure and heart rate. It also uses as a new method to measure blood pressure without unnecessary constriction of the patient's limb. They used the M2M technologies and GPS/GSM module, which operates usually under M2M platform. It makes use of a cellular phone for voice and data communications, a GPS receiver for location tracing.

Mobile robots are mostly used to investigate hazardous and dangerous environments where the risks for human operation exist. This robot can also be used to interact with human such as take care the elderly and doing household chores. In future smart cities, mobile robots can take over some tedious and time-consuming tasks.to help them as fast as possible. They have very short time to find the victims in any calamity situation; otherwise the likelihood of finding the victims still alive is nearly zero. In such a critical situation, technology can be used to support rescuers in different tasks. Intelligent mobile robots and cooperative multi- agent robotic systems are increasingly being used in many different ways to find and save the victims in a faster and more efficient way. The robot that can do such tasks is well known as rescue robot. Rescue robot is a robot that has been precisely designed to do rescuing jobs in situations that are hazardous for mankind to handle it, for instance rainstorms, collapsed buildings, obstructions, and dangerous substances.

Robotic systems are one of the key solutions for providing smart services. AMBUBOT is preferred for an immediate treatment using Automated External $\backslash$ Defibrillator (AED) to administer the victim within a few minutes after collapsing by facilitating various modes of operation from manual to autonomous functioning to save someone's life in the smart cities The prototype with biomedical sensors are used for monitoring the patient health continuously. The location of the patient can also be tracked in case of emergency via GSM. The concept of high tech machines that can serve the people well or relieve humans of tiresome chores has been an object of human imagination. It can be seen with many of today's occupations have been replaced by automation in order to help prevent manual handling injuries in the workplace.

The smart world is expected to involve ubiquitous sensing, computing, and communication to achieve comprehensive interconnections of physical perception, cyber interaction, social correlation, and cognitive thinking. Increasing population density in urban environments demands adequate provision of services and infrastructure. This explosion in city population will present major challenges including air pollution, traffic congestion, health concerns, energy and waste management. As an emerging platform for that domain, a mobile robot can be employed in order to facilitate the health care operation as a smart operating vehicle in smart cities. $\backslash$ Most rescuers occur shortly after the event of a calamity happens. In that event, human rescuers will organize the rescue planning to get out to the calamity areas, find the victims, and In contrast, a mobile robot would be able to travel throughout the environment and can put their position wherever its condition.

The concept of high-tech machines that can serve the people well or relieve humans of tiresome chores has been an object of human imagination. It can be seen with many of today's occupations have been replaced by automation in order to help prevent manual handling injuries in the workplace.

\section{EXISTING SYSTEM}

$>$ It takes more response time

$>$ In smart cities, due to traffic, sometimes ambulance will not reach the emergency spot on time, it may causes death. 
$>$ To overcome these problems, Ambulance robot is used.

\section{PROPOSED SYSTEM}

$>$ The proposed ambulance robot for smart cities provides the service of ambulance with AED to help someone having a cardiac arrest.

$>$ No need to wait for the ambulance to give first aid solutions.

$>$ An emergency message and current position of victim will be generated

$>$ Also, message can be sent to the family members regarding current situation of the victim.

$>$ It performs time-consuming tasks.

$>$ It can be operated in auto or manual mode.

\section{BLOCK DIAGRAM}

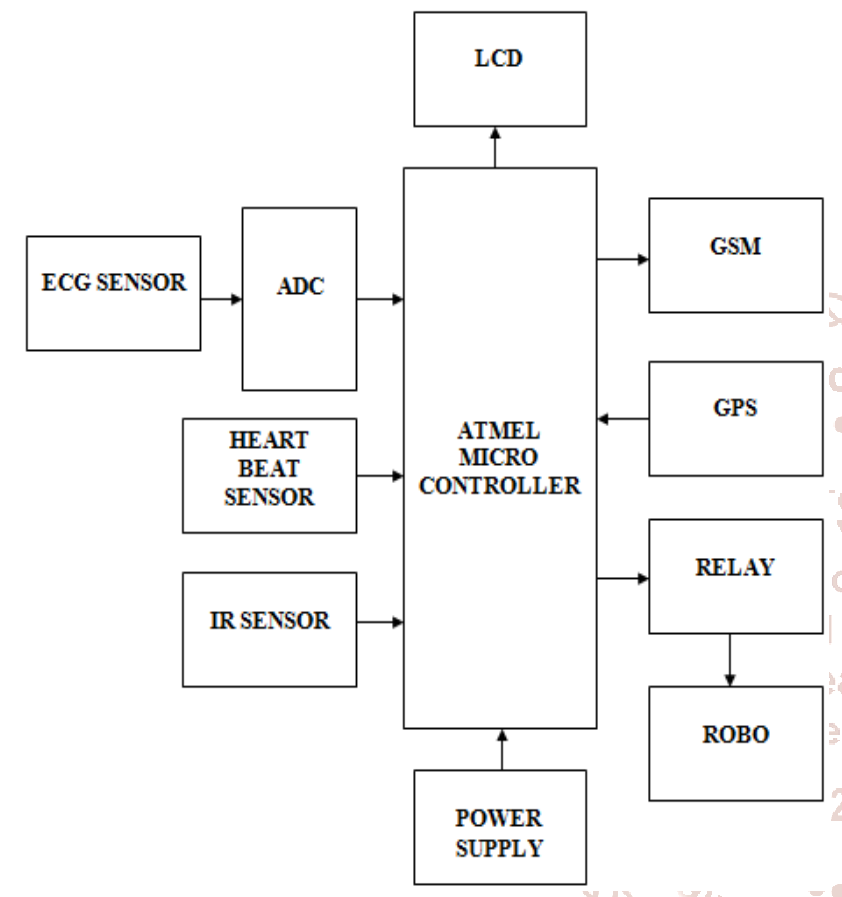

V. HARADWARE REQUIREMENTS

$>$ ATMEL MICROCONTROLLER

$>$ ECG SENSOR

$>$ HEART BEAT SENSOR

$>$ IR SENSOR

$>$ GSM

$>$ GPS

$>$ RELAY

$>$ ROBO

$>\mathrm{LCD}$

\section{LCD}

LCD (liquid crystal display) is the technology used for displays in notebook and other smaller computers. Like light-emitting diode (LED) and gas-plasma technologies, LCDs allow displays to be much thinner than cathode ray tube (CRT) technology. LCDs consume much less power than LED and gas-display displays because they work on the principle of blocking light rather than emitting it.

\section{GSM}

GSM (Global System for Mobile communications) is a standard developed by the European Telecommunications Standards Institute (ETSI) to describe the protocols for second-generation (2G) digital cellular networks used by mobile devices such as tablets. It was first deployed in
Finland in December 1991.[2] As of 2014, it has become the global standard for mobile communications - with over $90 \%$ market share, operating in over 193 countries and territories

\section{GPS}

A GPS navigation device, GPS receiver, or simply GPS is a device that is capable of receiving information from GPS satellites and then to calculate the device's geographical position. Using suitable software, the device may display the position on a map, and it may offer directions. The Global Positioning System (GPS) is a global navigation satellite system (GNSS) made up of a network of a minimum of 24, but currently 30 , satellites placed into orbit by the U.S. Department of Defense.

\section{RELAY}

A relay is a electromagnetic switch, It is used in application to turn on and of a circuit by low power signal $24 \mathrm{~V}$, or several circuits must be controlled by one signal.

\section{ROBOT}

A robot is a machine-especially one programmable by a computer - capable of carrying out a complex series of actions automatically.[2] Robots can be guided by an external control device or the control may be embedded within. Robots may be constructed to take on human form but most robots are machines designed to perform a task with no regard to how they look.

\section{POWER SUPPLY}

A power supply is an electrical device that supplies electric power to an electrical load. The primary function of a power supply is to convert electric current from a source to the correct voltage, current, and frequency to power the load.

\section{IR SENSOR}

An infrared sensor is an electronic device, that emits in order to sense some aspects of the surroundings. An IR sensor can measure the heat of an object as well as detects the motion. These types of sensors measures only infrared radiation, rather than emitting it that is called as a passive IR sensor.

\section{HEART BEAT SENSOR}

The heartbeat sensor is based on the principle of photo phlethysmography.... The flow of blood volume is decided by the rate of heart pulses and since light is absorbed by blood, the signal pulses are equivalent to the heart beat pulses.

\section{ADC}

In electronics, an analog-to-digital converter (ADC, A/D, or A-to-D) is a system that converts an analog signal, such as a sound picked up by a microphone or light entering a digital camera, into a digital signal. An ADC may also provide an isolated measurement such as an electronic device that converts an input analog voltage or current to a digital number representing the magnitude of the voltage or current. Typically the digital output is a two's complement binary number that is proportional to the input, but there are other possibilities.

\section{ATMEL MICRO CONTROLLER}

AVR is a family of microcontrollers developed since 1996 by Atmel, acquired by Microchip Technology in 2016. These are modified Harvard architecture 8-bit RISC single-chip microcontrollers. AVR was one of the first microcontroller families to use on-chip flash memory for program storage, as 
opposed to one-time programmable ROM, EPROM, or EEPROM used by other microcontrollers at the time.

\section{ECG SENSOR}

The Shimmer ECG (Electrocardiogram) sensor records the pathway of electrical impulses through the heart muscle, and can be recorded on resting and ambulatory subjects, or during exercise to provide information on the heart's response to physical exertion.

\section{SOFTWARE REQUIREMENTS \\ $>$ KEIL UVISION \\ $>$ EMBEDDED C}

\section{EMBEDDED C}

Embedded $\mathrm{C}$ is an extension to $\mathrm{C}$ programming language that provides support for developing efficient programs for embedded devices. It is not a part of the $C$ language.

\section{CONCLUSION}

In this paper, we presented a usage of smart vehicle for smart city which can be implemented to intelligent vehicles based on their architecture using a simple sequence of three steps: sense, plan, and act (SPA). Some of that technology has readily available on the market today and the rest of them are still on the investigation step to guarantee safe and reliable operation

\section{REFERENCES}

[1] H. Ning et al., "From Internet to smart world," IEEE Access, vol. 3, pp. 1994-1999, Oct. 2015.

[2] R. Jalali, K. El-khatib, and C. McGregor, "Smart city architecture for community level services through the Internet of Things," in Proc. IEEE 18th Int. Conf. Intell. Next Generat. Netw. (ICIN), Feb. 2015, pp. 108-113

[3] M. Arif, H. Samani, C.-Y. Yang, and Y.-Y. Chen, "Adaptation of mobile robots to intelligent vehicles," in Software Engineering and Service Science (ICSESS), 2013 4th IEEE International Conference on. IEEE,2013.

[4] F. Matsuno and S. Tadokoro, "Rescue robots and systems in japan," in Robotics and Biomimetics, 2004. ROBIO 2004. IEEE International Conference on. IEEE, 2004. [5] C.-P. Lam, C.-T. Chou, K.-H. Chiang and L.-C. $\mathrm{Fu}$, "Human-centered robot navigation towards a harmoniously human-robot coexisting environment, "Robotics, IEEE Transactions on, vol. 27, no. 1, pp. 99112, 2011.

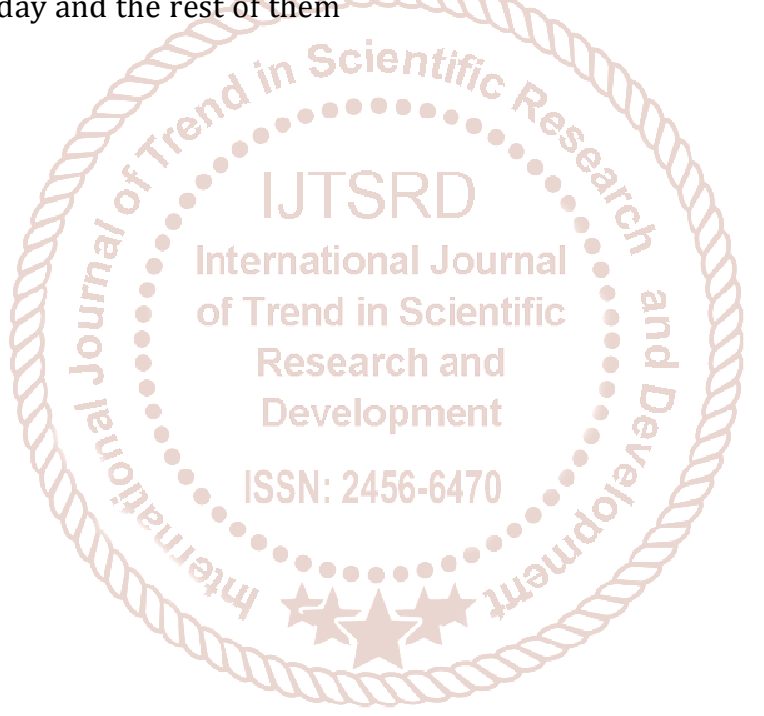

